\title{
Estudo das propriedades geotécnicas de resíduos sólidos urbanos recém-descartados e aterrados em aterro sanitário localizado na região Sul do Brasil \\ Study of the geotechnical properties of fresh and grounded municipal solid waste in a landfill located in Southern Brazil \\ Data de entrada: 28/08/2019 \\ Data de aprovação: 06/08/2020
}

Léa Beatriz Dai-Prá' | Luciana Paulo Gomes ${ }^{1 *}$ | Marcelo Oliveira Caetano' |

DOI: https://doi.org/10.36659/dae.2022.002 Pamela Lisie Ghesla ${ }^{1}$ | Marco Aurélio dos Santos ${ }^{1}$

ORCID ID

Dai-Prá LB (ID) https://orcid.org/0000-0002-5563-011X

Gomes LP (D) https://orcid.org/0000-0003-4542-1143
Caetano MO (D) https://orcid.org/0000-0002-0920-1971

Ghesla PL (D) https://orcid.org/0000-0002-3421-0407

Santos MA (D) https://orcid.org/0000-0002-8413-2275

\section{Resumo}

Aterros sanitários detêm a maior parcela de disposição final de Resíduos Sólidos Urbanos (RSU) no Brasil. O estudo das propriedades geotécnicas dos RSU contribui para o monitoramento das áreas de disposição, garante sua segurança estrutural e o aumento de vida útil, considerando a heterogeneidade dos resíduos. Este artigo apresenta os resultados do estudo das propriedades geotécnicas de uma amostra de RSU recémdescartados (1) e RSU aterrados há 5 anos (2), coletadas em um Aterro Sanitário localizado no Sul do Brasil. Foram realizados ensaios de teor de umidade, massa específica e compressão confinada, utilizando como metodologia a teoria de adensamento de solos. Com os ensaios de compressão, foram obtidos os valores de índice de compressão primária para a amostra $1(0,56$ a 1,67) e para a amostra $2(0,17$ a 0,53) e o índice de compressão secundária para amostra $2(0,006$ a 0,004). Este estudo confirmou a aplicabilidade da metodologia de solos para o estudo da compressibilidade de RSU.

Palavras-chave: Resíduos sólidos urbanos. Geotecnia. Adensamento. Ensaios laboratoriais.

\section{Abstract}

Sanitary landfills have the largest part of final disposal of Municipal Solid Waste (MSW) in Brazil. The study of the MSW geotechnical properties contributes to the monitoring of disposal areas, guarantees their structural safety and increases the useful life, considering the waste heterogeneity. This article presents the results of the study of geotechnical properties of a sample of MSW recently discarded (1) and MSW landed for 5 years (2), collected in a sanitary landfill located in southern Brazil. Tests of moisture content, specific density and confined compression were carried out, using the soil settlement theory as methodology. With the compression tests, the values of primary compression index were obtained for sample 1 (0.56 to 1.67) and for sample 2 (0.17 to 0.53) and secondary compression index for sample 2 (0.006 to 0.004). This study confirmed the applicability of the soil methodology for the study of MSW compressibility.

Keywords: Municipal solid waste. Geotechnics. Settlement. Laboratory tests.

\footnotetext{
${ }^{1}$ Universidade do Vale do Rio dos Sinos - São Leopoldo - Rio Grande do Sul - Brasil.

*Autora correspondente: lugomesaunisinos.br.
} 


\section{INTRODUÇÃO}

A disposição final de Resíduos Sólidos Urbanos (RSU) pode ser realizada por diversos métodos, alguns com valor monetário elevado, outros com maior custo-benefício, como é o caso dos aterros sanitários. No Brasil, essa tecnologia é utilizada por aproximadamente $74 \%$ dos municípios. (BRASIL, 2019).

Visto que os aterros sanitários detêm a maior parcela de destinação final dos RSU no Brasil, é importante salientar sua viabilidade técnica e econômica como sistema de disposição final, tanto para quem opera como para quem destina os resíduos. Porém essas áreas possuem uma vida útil de 20 a 30 anos apenas, sendo necessários estudos que visem a aumentar o tempo de utilização das mesmas. (DAI-PRÁ, 2018).

A compressibilidade dos resíduos durante a operação dos aterros, devido à decomposição da matéria orgânica, percolação de água da chuva, geração de lixiviado e compactação por máquinas e caminhões, deve ser estudada para prever o comportamento real do aterro sanitário, possibilitando a estimativa de estabilidade e vida útil. (DENARDIN, 2013).

Conforme ocorre a compressão dos resíduos pela operação do aterro, os materiais tendem a deformar, diminuir o volume e se deslocar horizontal e verticalmente. Com o passar do tempo, continua ocorrendo a deformação mecânica dos resíduos, enquanto a matéria orgânica e os materiais não inertes são decompostos. Para determinar os índices de compressão primária e secundária dos resíduos (Cc e C), que indicam o potencial de recalque que o aterro pode sofrer em função das diversas características de deformação dos materiais, têm sido empregados os conceitos da teoria clássica de adensamento de solos, com algumas adaptações, devido à dificuldade de quantificar os parâmetros necessários para es- tudar a compressibilidade dos RSU. (CARVALHO, VILAR e KAIMOTO, 2000; MACHADO et al., 2005).

Estudos sobre a compressibilidade dos RSU e ocorrência de recalques foram previamente desenvolvidos (Sowers, 1973; Carvalho, Vilar e Kaimoto, 2000; Nascimento, 2007; Reddy et al., 2009; Gomes e Caetano, 2010; Bareither, Benson e Edil, 2012; Shi et al., 2016, Dai-Prá, 2018). Vários destes comentam sobre a dificuldade de determinação da compressibilidade, devido às características distintas de cada tipo de resíduo, operação do aterro, local de disposição, entre outros fatores não controláveis.

\subsection{Objetivos}

Estudar as propriedades geotécnicas de amostras de RSU recém-descartadas e outras já dispostos no Aterro Sanitário da Companhia Riograndense de Valorização de Resíduos (CRVR), na cidade de São Leopoldo, Rio Grande do Sul, Brasil, por meio de ensaios de compressão confinada, realizados em laboratório.

\section{METODOLOGIA}

\subsection{Coleta de amostras e caracterização dos RSU}

A coleta da amostra 1 foi realizada na Central de Triagem, imediatamente após os caminhões de coleta descarregarem os resíduos no local. Posteriormente, foi realizada segregação, obtendo uma amostra de 1,2 kg e que possuía as mesmas características em termos de gravimetria indicadas nos estudos de composição gravimétrica executados por Gomes et al. (2014) e Ogliari (2015). Partiu-se, portanto, de uma amostra, dita 1, que representa a composição dos RSU que não foram triados e foram encaminhados posteriormente para o aterro sanitário em São Leopoldo/RS, Brasil. 
A amostra 2 foi coletada na primeira fase de aterramento, datada de 2011, ou seja, os resíduos estavam aterrados havia 5 anos, na época do estudo. A coleta dessa amostra foi realizada com o auxílio de uma escavadeira hidráulica, no aterro sanitário da CRVR, em São Leopoldo/RS, Brasil. $\mathrm{O}$ aterro iniciou suas atividades no ano de 2011, em uma área de disposição de resíduos de 60 hectares, com capacidade diária de disposição de 1000 t.dia $^{-1}$, com previsão inicial de encerramento para 2031. Foi realizada a caracterização da amostra coletada no aterro, baseada na NBR 10.007. (ABNT, 2004).

Os resíduos (amostras 1 e 2) foram classificados em Recicláveis (papel/papelão, plástico, embalagens longa vida, metais e vidros), Rejeitos (matéria orgânica - resíduos sólidos putrescíveis, contaminantes biológicos e contaminantes químicos); tecidos, isopor e outros materiais não recicláveis.

Após a caracterização dos resíduos, os mesmos foram triturados no moinho de facas, para a realização dos ensaios seguintes. A granulometria final das amostras correspondeu a, aproximadamente, $20 \mathrm{~mm}$, medida da peneira utilizada após o moinho.

\subsection{Teor de umidade}

Para a determinação de teor de umidade da Amostra 1, apenas a parcela de resíduos sólidos putrescíveis (material orgânico) foi seca a $65^{\circ} \mathrm{C}$, seguindo MAPA (2013), pois tratava-se de resíduos recém-descartados, com pouca umidade aparente. $O$ ensaio foi realizado em triplicata.

A Amostra 2 foi submetida ao ensaio de teor de umidade utilizando a metodologia indicada na NBR 6457 (ABNT, 1986), que emprega a secagem do resíduo a $105^{\circ} \mathrm{C}-110^{\circ} \mathrm{C}$, pois os resíduos já estavam aterrados havia 5 anos, gerando lixiviado e umidade visualmente maior. Foram utilizados os conceitos de base seca e base úmida. 0 ensaio foi realizado em triplicata.

\subsection{Massa específica}

Foi realizado o ensaio de massa específica para ambas as amostras, após a secagem em estufa no ensaio de teor de umidade.

Para a Amostra 1, o ensaio foi realizado no Picnômetro com bomba à vácuo, seguindo o procedimento da ABNT NBR 6508 (ABNT, 1984) e utilizando somente a parcela orgânica do resíduo.

O ensaio da Amostra 2 foi realizado no equipamento Picnômetro a gás Hélio, modelo AccuPyc ${ }^{\circledR}$ II 1340, da marca Micromeritics, utilizando a amostra na íntegra.

\subsection{Ensaios de compressão}

Os parâmetros de compressão primária - $\mathrm{C}_{\mathrm{c}}$ (Eq. 1), compressão secundária - $C_{\alpha}$ (Eq. 2) e índice de vazios $-e$ (Eq. 3), foram determinados por meio do ensaio de adensamento unidimensional, conforme NBR 12007 - MB 3336 (ABNT, 1990) e ASTM D 2435:2011 - D2435M. (ASTM, 2011).

$\mathrm{Cc}=e_{1}-e_{2} / \log _{\sigma 2}-\log \sigma 1$

$\left.\mathrm{C} \alpha=\Delta \mathrm{e} / \Delta \log _{t}\right)$

$\left(e=\frac{H}{H s}-1\right)$

Para a amostra 1, o dispositivo utilizado para os ensaios de compressão foi uma célula de adensamento de grandes dimensões $(310 \mathrm{~mm}$ de diâmetro e $255 \mathrm{~mm}$ de altura), construída em ferro fundido, adaptada da NBR 12.007. (ABNT, 1990). $\mathrm{O}$ dispositivo é composto de uma base rígida que contém um anel acoplado, para conter o corpo de prova, e um cabeçote móvel de carregamento. Em substituição à pedra porosa e membrana filtrante, utilizadas para compressão em solo, utili- 
zou-se areia média e manta geotêxtil de $0,7 \mathrm{~mm}$ da marca Maccaferri, modelo MacTex®.

Para aplicação de carga no corpo de prova da amostra 1, foi utilizado o equipamento de tração e compressão da marca EMIC, modelo DL 2000 Classe I, com erro menor que $0.5 \%$. A fim de atingir o máximo deslocamento vertical possível para compressão do resíduo, foi utilizado um cilindro sólido, de aço inoxidável, entre a célula de carga da máquina de compressão e o cabeçote da célula de adensamento. A carga foi aplicada de 0 até $2000 \mathrm{kgf}$ (aproximadamente $260 \mathrm{kPa}$ ), que é a limitação da máquina, a uma taxa constante de deslocamento de $0.08 \mathrm{~mm} / \mathrm{s}$. No total, foram realizados sete ensaios de compressão para a amostra 1 (Ensaios 1.1, 1.2, 1.3, 1.4, 1.5, 1.6, 1.7), na célula de adensamento apresentada na Fig. 1 . Não foi aplicada carga de pré-adensamento, pois o resíduo era recém-descartado, logo ainda não havia sido aterrado.

Para a amostra 2, o método também seguiu a NBR 12.007 (ABNT, 1990), porém o equipa- mento utilizado foi o clássico Oedômetro (Fig. 2), já utilizado para ensaios em solo. A norma foi adaptada para as condições das amostras de RSU, que foram utilizadas com umidade natural, conforme coleta, mas com os resíduos triturados. Foram aplicadas as cargas de 20, $40,80,160,320$ e $640 \mathrm{kPa}$. Foram realizados 2 ensaios (2.1 e 2.2). Antes da aplicação das cargas pré-definidas, foi aplicada a carga de pré-adensamento de 113,3 kPa durante 5 min (cálculo apresentado na Eq. 4), considerando o adensamento já ocorrido no aterro sanitário (aproximadamente 12 t de carga da escavadeira hidráulica), com o intuito de acomodar o resíduo. Esta também foi uma adaptação da NBR 12.007, já que na norma o que está citado é que seja aplicada uma pressão de $5 \mathrm{kPa}$ para solos resistentes e $2 \mathrm{kPa}$ para solos moles; este procedimento foi testado e não houve resultado positivo, portanto verificou-se a necessidade de acomodar o resíduo com a carga de pré-adensamento mencionada anteriormente.

Carga de pré - adensamento $=11,55 \frac{t}{\mathrm{~m}^{2}} * 1000 \frac{\mathrm{kgf}}{t} * 9,80665 \mathrm{~N} * \frac{1 \mathrm{kN}}{1000 \mathrm{~N}}=113,3 \mathrm{kPa}$

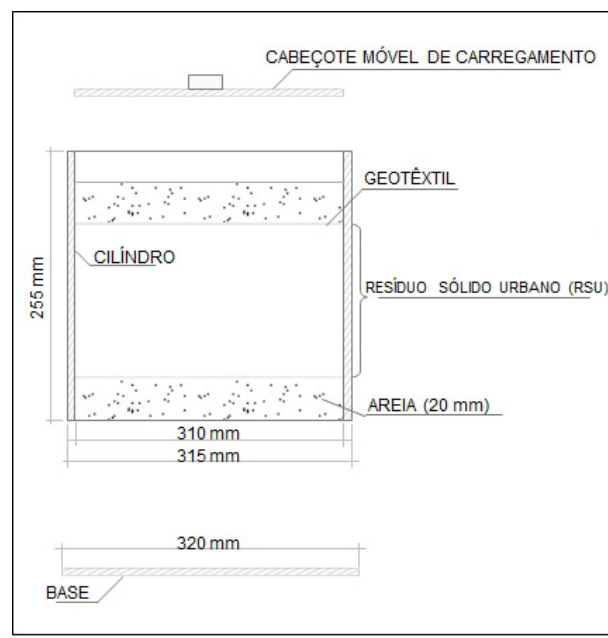

(a)

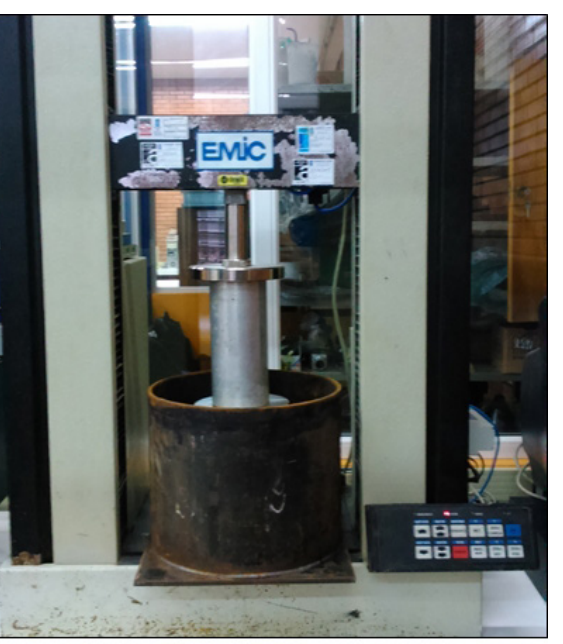

(b)

Figura 1 - Célula de adensamento empregada na amostra 1. (a) Esquema de representação da célula de adensamento; (b) Célula de adensamento em escala real 


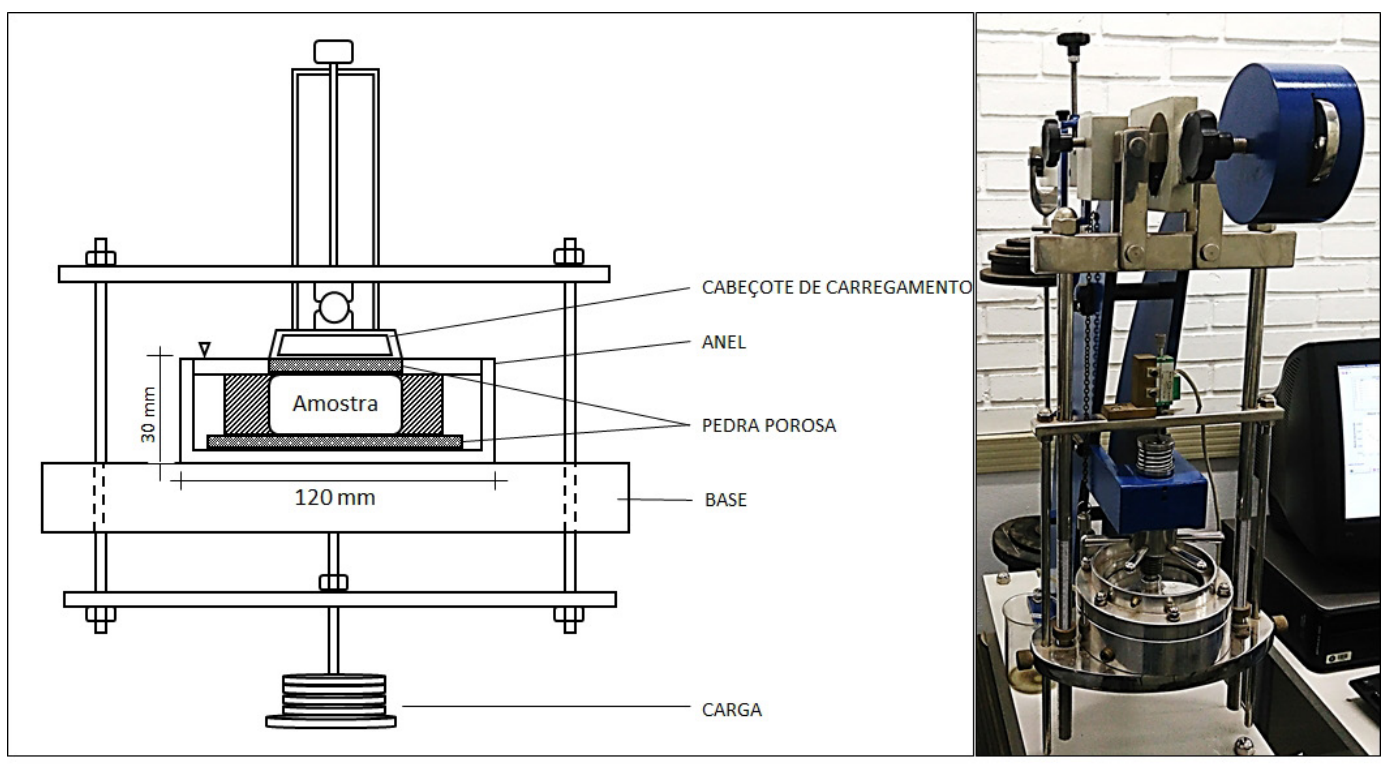

(a)

(b)

Figura 2 - Equipamento Oedômetro utilizado nos ensaios da amostra 2. (a) Esquema de representação do Oedômetro; (b) Oedômetro em escala real

A partir dos resultados dos ensaios foi possível obter os parâmetros de compressibilidade, bem como gráficos do comportamento dos RSU em função da aplicação de cargas.

\section{RESULTADOS E DISCUSSÃO}

\subsection{Caracterização gravimétrica}

A Fig. 3 apresenta a caracterização gravimétrica da amostra 1, e a Fig. 4 mostra a composição da amostra 2, de resíduos coletados no aterro.

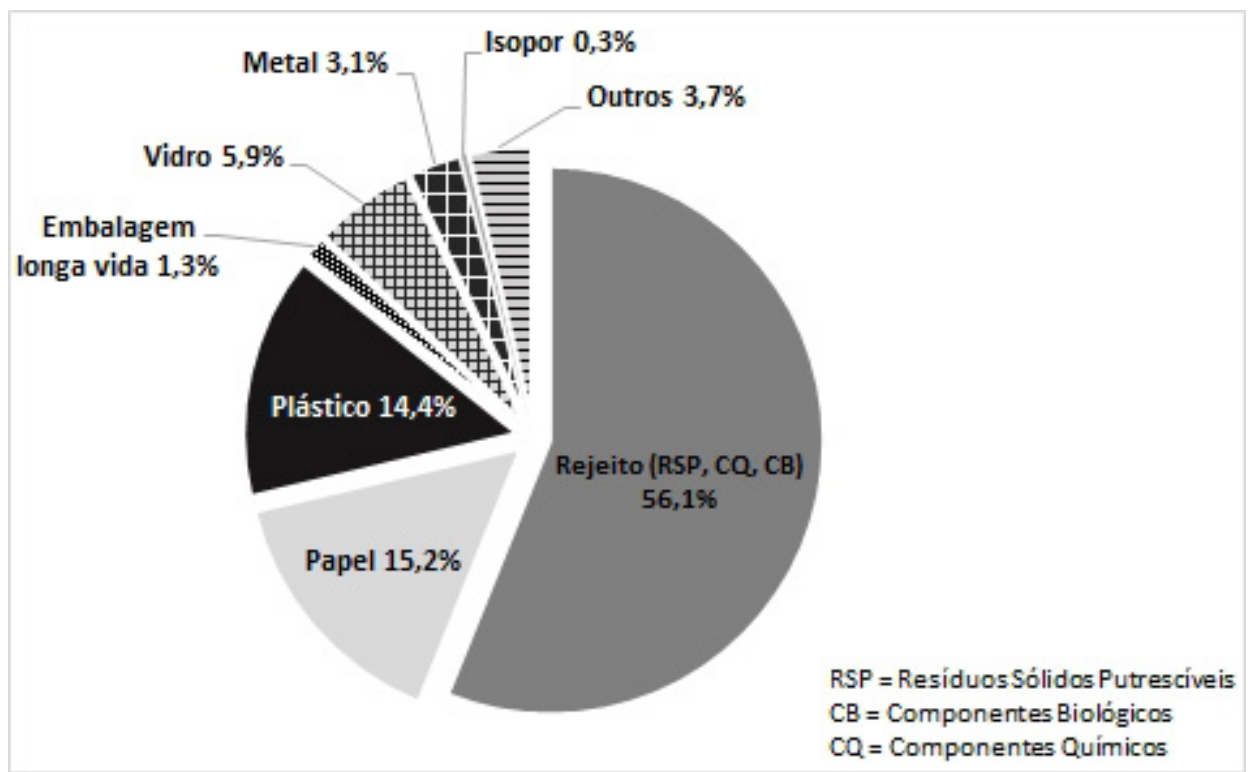

Figura 3 - Caracterização gravimétrica da amostra 1, resíduos novos 


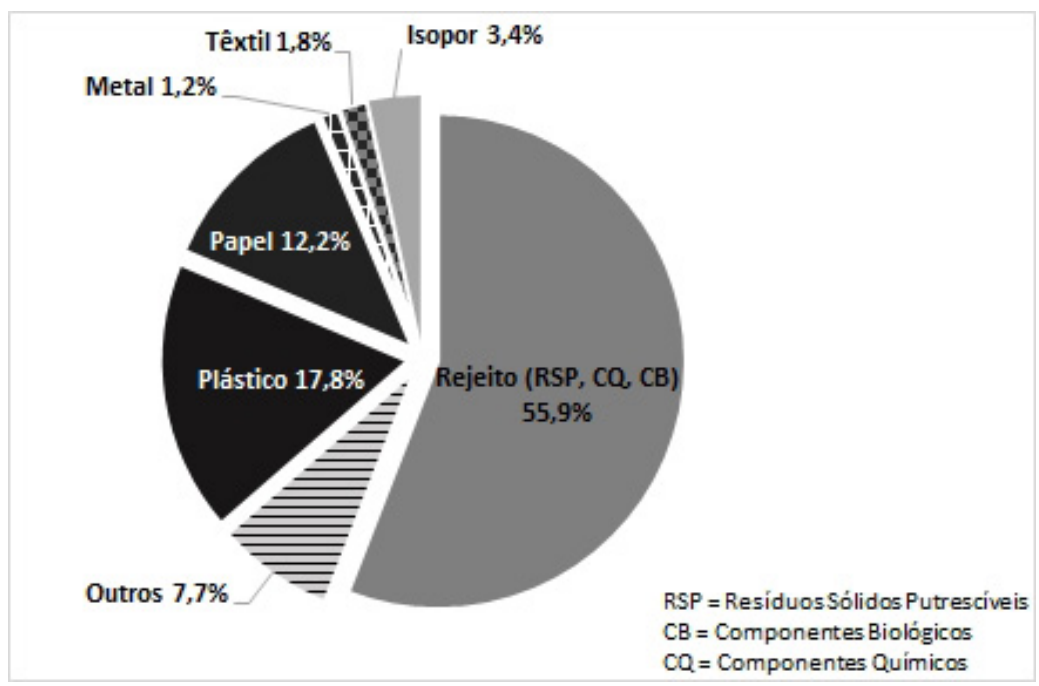

Figura 4 - Caracterização gravimétrica da amostra 2, resíduos aterrados

Verificou-se semelhança, principalmente quanto aos Plásticos, Papel e Metais, para ambas as amostras. O percentual de rejeitos quando somado ficou semelhante, porém, ressalta-se que a Amostra 1 possuía $45,5 \%$ de matéria orgânica (resíduos sólidos putrescíveis) e a Amostra 2 possuía 18,6\% da mesma categoria de resíduos. Portanto, foi verificado que o percentual de RSP realmente diminuiu, se comparada a amostra de resíduos novos (1) com a amostra de resíduos aterrados (2). Já a parcela "Outros” está relacionada principalmente a resíduos não identificados e outros materiais não recicláveis. As gravimetrias realizadas, exceto pelo percentual de RSP, mostram que as amostras eram similares, observando que ambas correspondem a resíduos gerados na mesma região Sul do Brasil. Considera-se, também, que a diferença nas categorias pode ser atribuída aos métodos de amostragem utilizados, que foram diferentes para cada amostra. As coletas foram pontuais, sendo que a Amostra 1 foi coletada imediatamente quando descartada e a Amostra 2 foi coletada com o auxílio de escavadeira hidráulica, superficialmente no aterro sanitário.

\subsection{Teor de umidade e massa específica}

O teor de umidade encontrado para a amostra 1 foi de $50,1 \%$ (base úmida), e para a amostra 2 foi de
157,0\% (base seca) e 61,0\% (base úmida). Verificouse que o teor de umidade da amostra 1 (50,1\%), de resíduos novos, está próximo aos valores apresentados por Reddy et al. (2009) e Nascimento (2007), que também utilizaram resíduos denominados novos, recém-descartados. Já para a amostra 2, de resíduos aterrados, em base seca $(157,0 \%)$ se assemelha ao apresentado por Marques, Filz e Vilar (2003), aterro com 4 anos de idade, e base úmida $(61,0 \%)$ assemeIha-se ao valor apresentado por Simões e Catapreta (2013), aterro com 6 anos de idade, ambos trabalhos desenvolvidos também no Brasil.

O valor de massa específica da amostra 1 (680 $\left.\mathrm{kg} . \mathrm{m}^{-3}\right)$ se assemelha ao valor obtido por Reddy et al. (2009) e por Marques (2001), resíduos estes com composição semelhante de materiais inertes. Para a amostra $2\left(1350 \mathrm{~kg} \cdot \mathrm{m}^{-3}\right)$, fica próximo da faixa proposta por Sowers (1973), na faixa de valores encontrados por Nascimento (2007), e relativamente próximo aos valores de Carvalho, Vilar e Kaimoto (2000), ambos com composição gravimétrica similar à amostra 2. Nota-se na literatura estudada, no caso de resíduos recémdescartados e aterrados, o mesmo fenômeno ocorrido neste estudo, onde o resíduo aterrado possui uma massa específica maior do que antes do aterramento. A heterogeneidade intrínseca 
dos resíduos é uma característica que influencia nos valores encontrados para cada estudo. É um motivo comentado pelos autores estudados, frisando que cada local tem suas particularidades e o valor de massa específica dos resíduos de um aterro não deve ser aplicado a outro.

\subsection{Ensaios de compressão}

Para a amostra 1, foram realizados sete ensaios de compressão, com carga de 0 a $260 \mathrm{kPa}$, a uma taxa constante de deslocamento de $0,08 \mathrm{~mm} / \mathrm{s}$. Os gráficos gerados a partir dos ensaios estão apresentados nas Fig. 5 e 6.
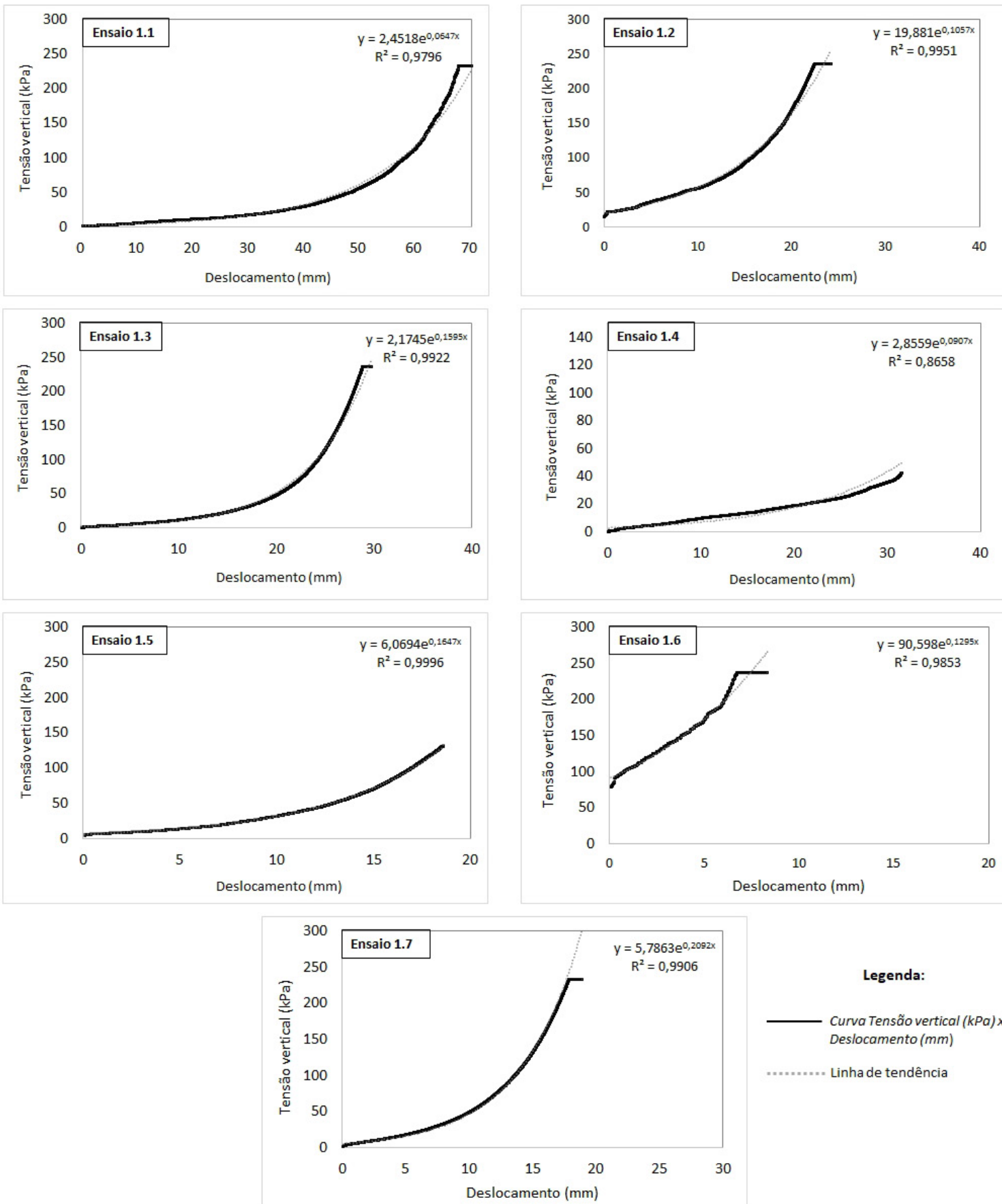

Legenda:

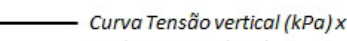
Deslocamento $(\mathrm{mm})$

Linha de tendência

Figura 5 -Curvas típicas de Tensão vertical $(\mathrm{kPa})$ versus deslocamento $(\mathrm{mm})$, amostra 1 

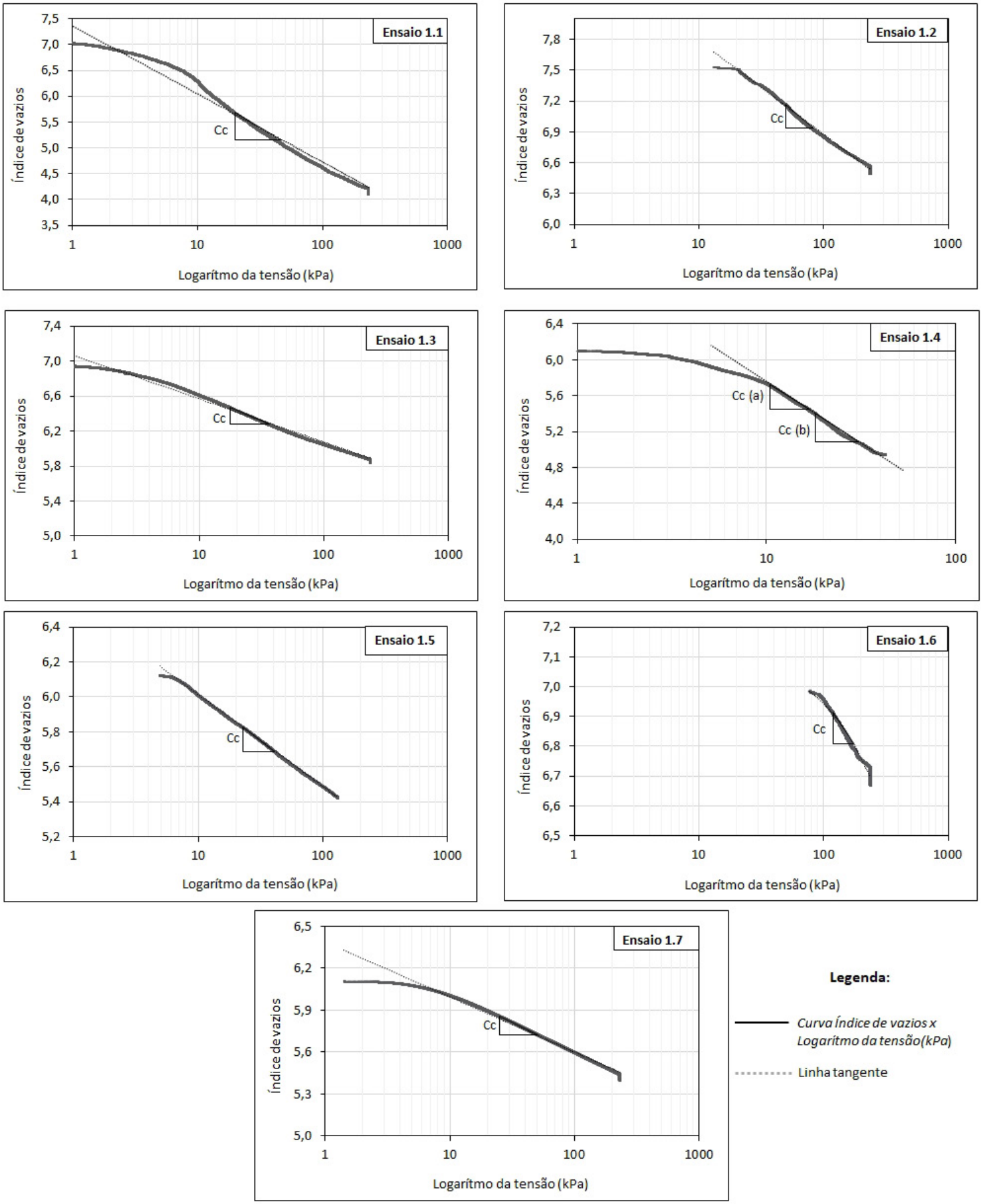

Legenda:

Curva índice de vazios $x$ Logaritmo da tensão( $\mathrm{kPa}$

Linha tangente

Figura 6- Relação Índice de vazios versus Logaritmo da tensão, amostra 1

Para a amostra 2, foram realizados dois ensaios de compressão completos, com o incremento de cargas de 20 a $640 \mathrm{kPa}$. A partir dos ensaios foi possível construir os gráficos de "deforma- ção versus tempo", "índice de vazios versus raiz do tempo", "índice de vazios versus logaritmo do tempo", e "índice de vazios versus logaritmo da tensão", apresentados nas Fig. 7, 8, 9 e 10. 

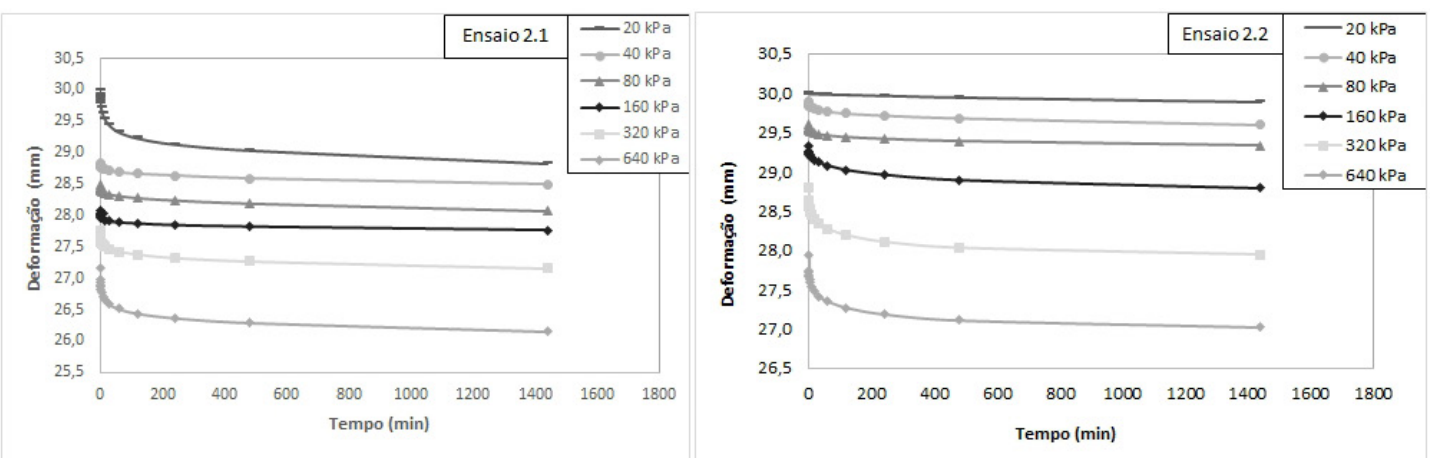

Figura 7 - Gráficos Deformação x Tempo, amostra 2

Pelo comportamento dos gráficos apresentados na Fig. 7, verifica-se que no carregamento de 20 $\mathrm{kPa}$ ainda ocorria o acomodamento do resíduo, mesmo tendo considerado a carga de pré-adensamento. Como nesses ensaios, que são baseados na metodologia de solos, ocorrem adaptações, possivelmente o tempo de $5 \mathrm{~min}$ para pré-adensar o resíduo não foi suficiente, sendo o restante acomodado nos carregamentos iniciais, principalmente no de $20 \mathrm{kPa}$, para ambos os en-

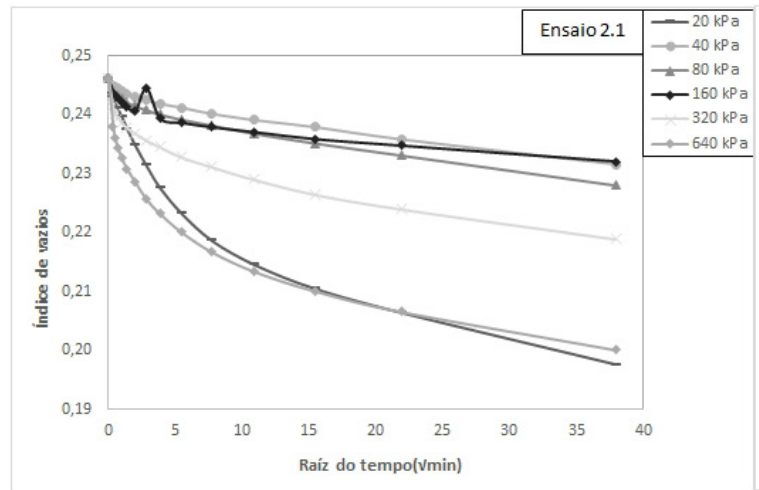

saios. Sendo assim, desconsiderou-se o carregamento de $20 \mathrm{kPa}$ para os cálculos dos parâmetros de compressibilidade, dada sua instabilidade.

Pode-se observar nos gráficos da Fig. 8 que a compressão primária, caracterizada pela curvatura mais acentuada, ocorre nos primeiros minutos de ensaio, sendo que o restante das deformações é caracterizado pela compressão secundária, pelo aumento do comportamento linear.

Figura 8 - Gráficos Índice de vazios x Raiz do tempo, amostra 2

Na Fig. 9, o comportamento dos gráficos de índice de vazios versus o logaritmo do tempo (min) evidencia o processo de compressão se-

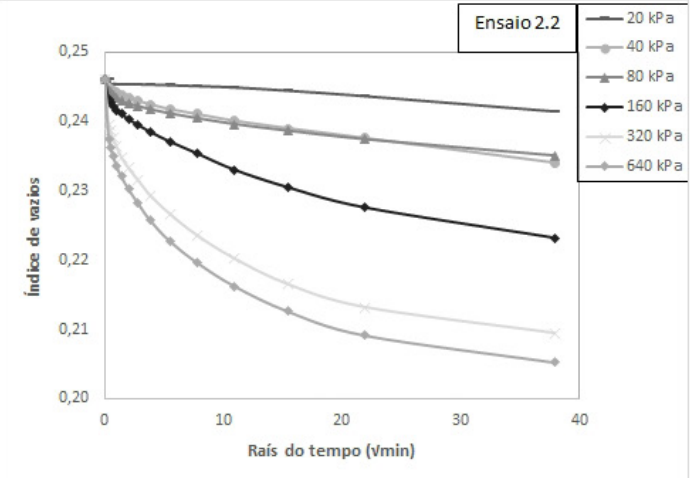



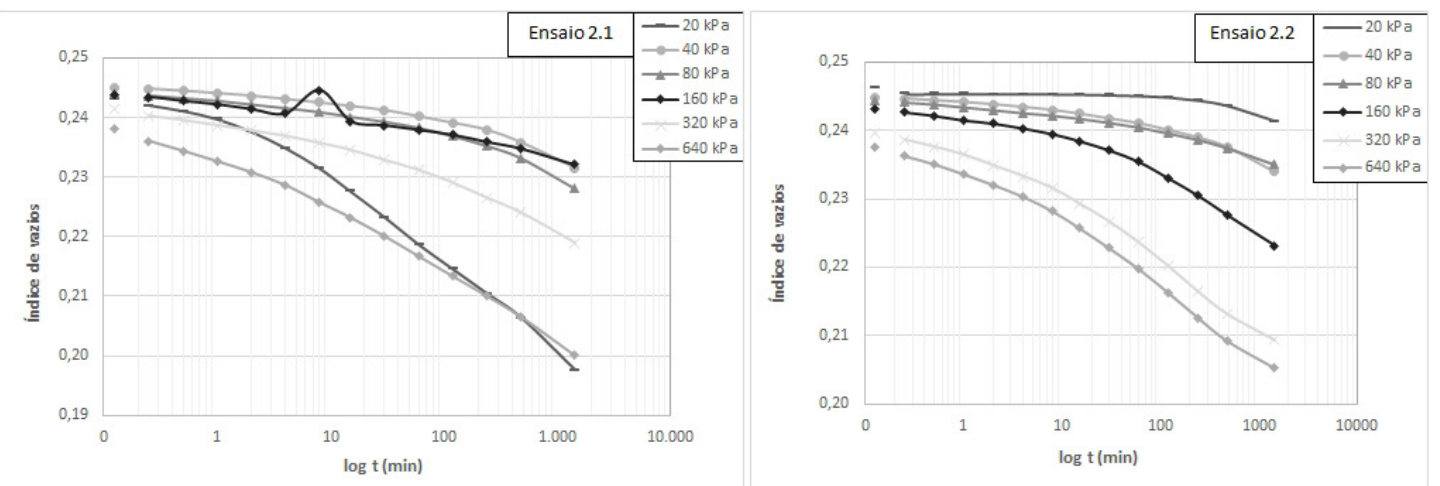

Figura 9 - Gráficos Índice de vazios x Logaritmo do tempo, amostra 2

Nas Fig. 8 e 9 observa-se comportamento semeIhante aos gráficos apresentados por Carvalho, Vilar e Kaimoto (2000), Nascimento (2007) e Shi et al. (2016), que realizaram estudos similares quanto ao comportamento dos resíduos em en- saios de compressão, utilizando a metodologia de mecânica dos solos.

A Fig. 10 apresenta o cruzamento dos dois ensaios no gráfico de índice de vazios versus o logaritmo da pressão $(\mathrm{kPa})$.

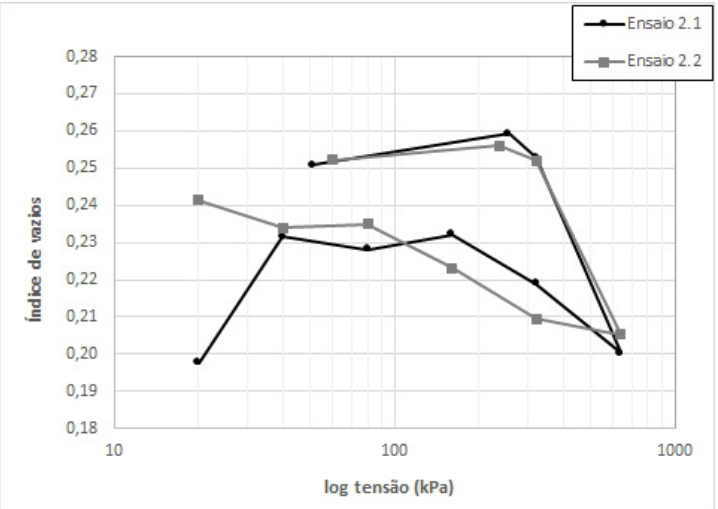

Figura 10 - Gráfico Índice de vazios x logaritmo da pressão, amostra 2

Conforme pode ser observado na Fig. 10, nas primeiras cargas aplicadas, os resíduos sofreram variações no índice de vazios, possivelmente devido ao leve acomodamento do material durante esses estágios de pressão. Posteriormente, a partir de $80 \mathrm{kPa}$ para o ensaio 2.1, e $160 \mathrm{kPa}$ para o ensaio 2.2, o formato das curvas se adequou ao esperado, apresentando o decréscimo do índice de vazios conforme aumentou a pressão aplicada e, quando reduzida a pressão, o índice de vazios novamente aumentou, apresentando comportamento semeIhante aos estudos de Nascimento (2007) e CarvaIho, Vilar e Kaimoto (2000), trabalhos que também estudaram resíduos sólidos urbanos aterrados coletados em aterros de grande porte.

É importante frisar que o trecho virgem das curvas na Fig. 10, conforme a teoria de mecânica dos solos, inicia-se após a tensão de pré-adensamento destes ensaios $(113,3 \mathrm{kPa})$, pois é o trecho que começa a partir de uma pressão que o resí- 
duo ainda não sofreu. Vale ressaltar que, dado o tipo de amostra, qualquer ensaio pode apresentar características diferentes entre si, principalmente pela heterogeneidade dos resíduos. Por meio dessa curva foi possível determinar os ín- dices e os coeficientes de compressão primária e secundária (Cc, C'c, C'a, C'a).

A Tabela 1 apresenta os parâmetros de compressibilidade obtidos nos ensaios da amostra 1 , em comparação à literatura estudada.

Tabela 1 - Compressibilidade da amostra 1, resíduos novos

\begin{tabular}{|c|c|c|c|c|c|c|}
\hline Autor & Observação & $\omega(\%)$ & $\mathbf{e}_{0}$ & e & $\mathrm{C}_{\mathrm{c}}$ & $C_{c}^{\prime}$ \\
\hline Este estudo & Valores médios (resíduos novos) & $\begin{array}{l}157 \text { (b.s.) } \\
61 \text { (b.u.) }\end{array}$ & $\begin{array}{l}7,115- \\
7,998\end{array}$ & $\begin{array}{c}4,099- \\
6,672\end{array}$ & $\begin{array}{l}0,561- \\
1,666\end{array}$ & $\begin{array}{c}0,069- \\
0,205\end{array}$ \\
\hline Sowers (1973) & Valores sugeridos (estudo em vários aterros) & $\begin{array}{l}10-50 \\
\text { (b.u.) }\end{array}$ & - & $\begin{array}{l}2,000- \\
15,000\end{array}$ & $\begin{array}{l}0,150 \mathrm{e} 0- \\
0,550 \mathrm{e} 0\end{array}$ & - \\
\hline $\begin{array}{l}\text { Carvalho, Vilare } \\
\text { Kaimoto (2000) }\end{array}$ & $\begin{array}{c}\text { Resíduos aterrados há } 15 \text { anos - Aterro Sanitário } \\
\text { Bandeirantes, São Paulo/SP - Brasil }\end{array}$ & 95,4 (b.u.) & 1,228 & 3,353 & 0,890 & 0,204 \\
\hline Nascimento (2007) & $\begin{array}{l}\text { Resíduos novos - Aterro Metropolitano Centro Salvador/ } \\
\text { BA - Brasil }\end{array}$ & 135 (b.s.) & 4,134 & - & 1,459 & 0,284 \\
\hline Reddy et al. (2009) & Resíduos novos - Aterro Orchard Hills, EUA & 70 (b.s.) & - & - & 0,270 & - \\
\hline Shi et al. (2016) & $\begin{array}{l}\text { Amostra preparada - resíduos novos (composição } \\
\text { gravimétrica da China) }\end{array}$ & - & 4,000 & - & 0,349 & - \\
\hline
\end{tabular}

Legenda: $\omega$ (\%) - teor de umidade; $e_{0}$ - Índice de vazios inicial; e-Índice de vazios; Cc -Índice de compressão primária; C'c-Coeficiente de compressão primária; b.s. - base seca; b.u. - base úmida.

Em relação aos resultados da amostra 1, em comparação com a literatura estudada, considera-se plausível a diferença encontrada para alguns parâmetros. As condições de ensaio e as características dos resíduos, em comparação aos autores analisados, foram divergentes em aspectos como o equipamento de compressão e condições de coleta, e o mais importante, o RSU era novo, enquanto alguns estudos utilizaram RSU aterrado. Possivelmente, foram estas as causas das diferenças entre os valores obtidos.

Carvalho, Vilar e Kaimoto (2000) obtiveram valor de índice de vazios inicial médio de 3,353 e, após o ensaio de compressão, valor médio de 1,228. Os autores utilizaram amostras aterradas há 15 anos e ressaltam a dificuldade em determinar o índice de vazios dos corpos de prova. Da mesma forma, Nascimento (2007) obteve valor de índice de vazios médio no início do carregamento, para resíduos novos de 4,134. Shi et al. (2016) também determinaram o índice de vazios inicial de resíduos novos e obtiveram um valor médio de
4,000. O valor médio obtido neste estudo para o índice de vazios ficou, também, na faixa sugerida por Sowers (1973).

O índice de compressão primária (Cc) determinado neste trabalho encontra-se abaixo, mas próximo, do determinado por Nascimento (2007), e acima dos valores obtidos por Reddy et al. (2009) e Shi et al. (2016). Esse resultado indica que o índice de vazios influencia diretamente nesse parâmetro, pois quanto maior é o índice de vazios, maior será o Cc, levando sempre em consideração as características intrínsecas de cada amostra e tipo de resíduo. O coeficiente de compressão primária ( $\left(C^{\prime} c\right)$ obtido ficou próximo aos valores obtidos por Carvalho, Vilar e Kaimoto (2000) e Nascimento (2007).

A Tabela 2 apresenta os índices de compressibilidade obtidos por meio dos cálculos e gráficos gerados a partir dos ensaios de adensamento da amostra 2, coletada no aterro sanitário da CRVR. 
Tabela 2 - Compressibilidade da amostra 2, resíduos aterrados (5 anos)

\begin{tabular}{|c|c|c|c|c|c|c|c|}
\hline Autor & Observação & $\mathbf{e}_{0}$ & e & $\mathrm{C}_{\mathrm{c}}$ & $C_{c}^{\prime}$ & $\mathrm{C}_{\alpha}$ & $C_{\alpha}^{\prime}$ \\
\hline \multirow[t]{2}{*}{ Este estudo } & Ensaio 1 & 0,246 & $\begin{array}{l}0,224- \\
0,241\end{array}$ & 0,170 & 0.140 & 0,006 & 0,005 \\
\hline & Ensaio 2 & 0,246 & $\begin{array}{c}0,226- \\
0,245\end{array}$ & 0,530 & 0.430 & 0,004 & 0,003 \\
\hline Sowers (1973) & Valores sugeridos (estudo em vários aterros) & - & $\begin{array}{l}2,000- \\
15,000\end{array}$ & $\begin{array}{c}0,150 \mathrm{e} 0- \\
0,550 \mathrm{e} 0\end{array}$ & - & $\begin{array}{l}0,030 e_{0}- \\
0,090 e_{0}\end{array}$ & - \\
\hline $\begin{array}{l}\text { Bjarngard; Edgers } \\
\text { (1990) }\end{array}$ & Laboratório e campo (dados de 24 aterros) & - & - & $\begin{array}{c}0,050- \\
0,260\end{array}$ & - & $\begin{array}{c}0,003- \\
0,056\end{array}$ & - \\
\hline $\begin{array}{l}\text { Carvalho, Vilare } \\
\text { Kaimoto (2000) }\end{array}$ & $\begin{array}{c}\text { Resíduos aterrados há } 15 \text { anos - Aterro Sanitário } \\
\text { Bandeirantes, São Paulo/SP - Brasil }\end{array}$ & 1,228 & 3,353 & 0,89 & 0.204 & 0,032 & 0,013 \\
\hline Nascimento (2007) & $\begin{array}{c}\text { Resíduos novos - Aterro Metropolitano Centro } \\
\text { Salvador/BA - Brasil }\end{array}$ & 4,134 & - & 1,459 & 0.284 & 0,079 & 0,010 \\
\hline Reddy et al. (2009) & Resíduos novos - Aterro Orchard Hills, EUA & - & - & 0,27 & - & - & 0,024 \\
\hline Shi et al. (2016) & $\begin{array}{l}\text { Amostra preparada - resíduos novos } \\
\text { (composição gravimétrica da China) }\end{array}$ & 4,000 & & 0,349 & & $\begin{array}{l}0,034- \\
0,049\end{array}$ & - \\
\hline
\end{tabular}

Legenda: $e_{0}$ - Índice de vazios inicial; e - Índice de vazios; $C c$ - Índice de compressão primária; C'c [C'c=Cc/(1 + e e $)$ - Coeficiente de compressão primária; $\mathrm{C} \alpha$-Índice de compressão secundária.

Analisando os valores obtidos para a amostra 2, em comparação com a literatura, verifica-se que o índice de vazios inicial e final está abaixo dos valores apresentados pelos autores estudados. Conforme confirmado por Bjarngard e Edgers (1990), Carvalho, Vilar e Kaimoto (2000) e Nascimento (2007), esse parâmetro é de difícil obtenção, dada a heterogeneidade dos resíduos.

Entre os ensaios realizados, nota-se uma diferença nos valores de índice e coeficiente de compressão primária (Cc e C'c), mesmo se tratando da mesma amostra, confirmando a influência da heterogeneidade dos resíduos. Ainda assim, a faixa de valores obtida para a amostra analisada encontra-se dentro da faixa proposta por Sowers (1973), e próxima aos valores obtidos por CarvaIho, Vilar e Kaimoto (2000), Reddy et al. (2009) e Shi et al. (2016).

Os valores de índice e coeficiente de compressão secundária ( $\mathrm{C} \alpha$ e C' $\alpha$ ) ficaram abaixo dos valores obtidos pelos autores analisados na Tabela 2, exceto para Bjarngard e Edgers (1990), em que os valores ficaram dentro da faixa obtida pelos autores. Como o equipamento utilizado somente realiza ensaios de $24 \mathrm{~h}$ para cada carga aplicada, possivelmente o tempo de ensaio não foi suficiente para prever a compressão secundária, que ocorre em longo prazo. Assim, a compressão primária pôde ser medida, pois ocorreu nos primeiros minutos de ensaio, mas a compressão secundária, se houvesse a possibilidade, poderia ter sido ensaiada por mais tempo, para garantir os resultados em longo prazo. Mesmo assim, o comportamento da compressibilidade se confirma pelo delineamento das curvas apresentadas anteriormente, o que mostra que, apesar de em alguns casos os valores obtidos não serem similares aos autores estudados, cada tipo de RSU possui suas características de teor de umidade e massa específica, clima da região onde estão aterrados, operação do aterro, entre outros fatores que tornam cada local de estudo único, mesmo tratando-se todos de estudos com resíduos sólidos urbanos.

\section{CONCLUSÃO}

Para este estudo, foram considerados dois tipos de amostra de RSU, uma sem aterramento (Amostra 1) e outra já aterrada há 5 anos (Amostra 2). Foram observadas diferenças entre as amostras, devido a vários fatores como amostragem, condições de ensaio e características intrínsecas dos resíduos (heterogeneidade, teor de umidade, 
massa específica, etc.). 0 índice de vazios final para a amostra 1, por exemplo, foi determinado na faixa de 4,099-6,672, valores aproximados aos dados apresentados por autores clássicos da geotecnia de resíduos. Já na amostra 2, os valores ficaram na faixa de 0,224-0,245 (média dos ensaios), abaixo da média apresentada pelos autores estudados, onde estes salientam que é um parâmetro de difícil obtenção, considerando a heterogeneidade dos resíduos.

O índice de compressão primária (Cc) obtido para a amostra 1 foi na faixa de $0,561-1,666$, o que confirma a influência do índice de vazios inicial (7,115 - 7,998) na determinação deste parâmetro; mesmo assim, o coeficiente ficou próximo dos valores obtidos nos estudos de Carvalho, Vilar e Kaimoto (2000) e de Nascimento (2007), ambos estudos clássicos da teoria de adensamento de resíduos. Já o Cc para a amostra 2 ficou na faixa de 0,170 0,530, dentro da faixa proposta por Sowers (1973), e próximo aos valores obtidos por Carvalho, Vilar e Kaimoto (2000), Reddy et al. (2009) e Shi et al. (2016). Para a amostra 2, determinou-se também o índice de compressão secundária $(C \alpha)$, que ficou na faixa de 0,006 0,004 , abaixo dos valores obtidos pela maioria dos autores estudados, exceto para Bjarngard e Edgers (1990), em que os valores ficaram dentro da faixa obtida.

Verificou-se a importância da realização de estudos de compressibilidade com resíduos recémdescartados e também dos já aterrados, pois ambas as condições influenciam diretamente na obtenção dos índices; principalmente no coeficiente de compressão primária, que é diretamente proporcional ao índice de vazios. Ressalta-se que a composição dos resíduos deve ser levada sempre em consideração em todos os estudos de compressibilidade, já que qualquer mudança nas características dos resíduos pode influenciar nos resultados finais de compressão.

\section{CONTRIBUIÇÃO DOS AUTORES}

Todos os autores contribuíram de forma igualitária.

\section{REFERÊNCIAS}

ASSOCIAÇÃO BRASILEIRA DE NORMAS TÉCNICAS (ABNT). ABNT NBR 10.007: Amostragem de resíduos sólidos. Rio de Janeiro, 2004.

ABNT NBR 12007: Solo - Ensaio de adensamento unidimendional. Rio de Janeiro, 1990.

ABNT NBR 6457: Amostras de solo - Preparação para ensaio de compactação e ensaios de caracterização. Rio de Janeiro, 1986.

ABNT NBR 6508: Grãos de solo que passam na peneira de 4,8 mm - Determinação da massa específica. Rio de Janeiro, 1984.

AMERICANSOCIETYFOR TESTING AND MATERIALS (ASTM). ASTM D 2435:2011 D2435M: Standard Test Methods for One-Dimensional Consolidation Properties of Soils Using Incremental Loading. West Conshokocken/PA, 2011.

BAREITHER, C. A.; BENSON, C. H.; EDIL, T. B. Compression Behavior of Municipal Solid Waste: Immediate Compression. Journal of Geotechnical and Geoenvironmental Engineering, v. 138, n. 9, p. 1047-1062, 2012. https://doi.org/10.1061/(ASCE)GT.19435606.0000672

BJARNGARD, A.; EDGERS, L. Settlements of municipal solid waste Landfills. 13th Annual Madison Waste Conference. Anais... Madison: 1990

BRASIL. Diagnóstico do Manejo de Resíduos Sólidos Urbanos 2018. Brasília, Ministério das Cidades, Secretaria Nacional de Saneamento Ambiental, Sistema Nacional de Informações sobre Saneamento - SNIS, 2019. Disponível em: <http://www.snis.gov.br/ diagnostico-anual-residuos-solidos/diagnostico-do-manejo-deresiduos-solidos-urbanos-2018>. Acesso em: 11 maio 2020.

CARVALHO, M. F.; VILAR, O. M.; KAIMOTO, L. S. A. Estudo de Compressibilidade de Resíduos Sólidos Urbanos. Solos e Rochas: Revista Latino-americana de Geotecnia, v. 23, n. 1, p. 2000.

DAI-PRÁ, L. B. Desenvolvimento e aplicação de um modelo de predição de recalques no aterro sanitário da CRVR - unidade de São Leopoldo/RS. Dissertação (Mestrado em Engenharia Civil), Programa de Pós-Graduação em Engenharia Civil, Universidade do Vale do Rio dos Sinos - Unisinos. São Leopoldo, 2018.126 p.

DENARDIN, G. P. Estudo dos recalques do aterro sanitário da Central de Resíduos do Recreio - Minas do Leão/RS. Dissertação (Mestrado em Engenharia Civil). Universidade Federal de Santa Maria (UFSM). Santa Maria, 2013.

GOMES, L. P.; CAETANO, M. O. \& MIRANDA, L. A. S. Ammoniacal nitrogen removal from sanitary landfill leachate by stripping process using a baffled reactor. Revista AIDIS de Ingeniería y Ciencias 
Ambientales: Investigación, desarrolloy práctica, 7(1): 19-32, 2014. http://dx.doi.org/10.22201/iingen.0718378xe.2014.7.1.46610

MACHADO, S. L.; CARVALHO, M. F.; DOURADO, K. A.; ROCHA, M. B.; LOPES, C. L. Estudo da compressibilidade de resíduos sólidos urbanos da região metropolitana de Salvador -BA. 23o Congresso Brasileiro de Engenharia Sanitária e Ambiental. Anais eletrônicos... Campo Grande, MS: ABES - Associação Brasileira de Engenharia Sanitária e Ambiental, 2005

MARQUES, A. C. M. Compactação e compressibilidade de resíduos sólidos urbanos. Tese (Doutorado em Geotecnia), Escola de Engenharia de São Carlos, Universidade de São Paulo (USP). São Carlos, 2001. 408p.

MARQUUES, A. C. M.; FILZ, G. M.; VILAR, O. M. Composite Compressibility Model for Municipal Solid Waste. Journal of Geotechnical and Geoenvironmental Engineering, v. 129, n. 4, p. 372-378, 2003. https://doi.org/10.1061/(ASCE)10900241(2003)129:4(372)

MINISTÉRIO DA AGRICULTURA PECUÁRIA E ABASTECIMENTO (MAPA). Manual de métodos analíticos oficiais para fertilizantes e corretivos. Brasília, 2013.141 p.

NASCIMENTO, J. C. F. Comportamento mecânico de resíduos sólidos urbanos. Dissertação (Mestrado em Geotecnia), Universidade de São Paulo, São Carlos, 2007.
OGLIARI, E. M. (2015). Avaliação de duas cooperativas de catadores de resíduos sólidos urbanos para identificação de parâmetros operacionais e de gerenciamento que influenciam na quantidade de rejeitos gerados no processo de coleta e triagem. Dissertação (Mestrado e m Engenharia Civil). Universidade do Vale do Rio dos Sinos (UNISINOS), São Leopoldo, $128 \mathrm{p}$.

REDDY, K. R.; HETTIARACHCHI, H.; PARAKALLA, N. S.; GANGATHULASI, J.; BOGNER, J. E. Geotechnical properties of fresh municipal solid waste at Orchard Hills Landfill, USA. Waste Management, v. 29, n. 2, p. 952-959, 2009. https://doi.org/ 10.1016/j.wasman.2008.05.011

SHI, J. QIAN, X.; LIU, X.; SUN, L.; LIAO, Z. The behavior of compression and degradation for municipal solid waste and combined settlement calculation method. Waste Management, v. 55, p. 154-164, 2016. https://doi.org/10.1016/j.wasman.2015.09.035

SIMÕES, G. F.; CATAPRETA, C. A. A. Monitoring and modeling of long-term settlements of an experimental landfill in Brazil. Waste Management, v. 33, n. 2, p. 420-430, 2013. https://doi. org/10.1016/j.wasman.2012.10.001

SOWERS, G. F. Settlement of waste disposal fills. 8th International Conference on Soil Mechanics and Foundation Engineering. Anais... Moscow, 1973. 